\title{
Kahoot! como herramienta para mejorar los resultados académicos en educación superior
}

\author{
Martínez Jiménez, Rocío ${ }^{\mathrm{a}}$, Ruiz Jiménez, $\mathbf{M}^{\mathrm{a}}$ Carmen $^{\mathrm{b}}$; García Martí, Elia ${ }^{\mathrm{c}}$; Pedrosa Ortega, \\ Cristina $^{\mathrm{d}}$ y Licerán Gutiérrez, Ana $^{\mathrm{e}}$ \\ Universidad de Jaén \\ Contactos: ${ }^{a}$ rmartine@ujaen.es, ${ }^{b}$ cruiz@ujaen.es; ${ }^{c}$ egarcia@ujaen.es; ${ }^{\mathrm{d}}$ cpedrosa@ujaen.es; ${ }^{\mathrm{e} a l i c e r a n @ u j a e n . e s ~}$
}

\begin{abstract}
The use of more active and participative methodological tools in the classroom is a growing trend in any university. In addition, we observe how it is increasingly common to use smartphones or tablets that allow gamification with students through applications such as Kahoot!. Therefore, several works have shown some positive effects of this tool, such as improving the students' interest, attention and motivation. However, very few studies have analyzed whether the use of this Kahoot! tool positively influences students' academic performance.

In this paper we present the results of a gamification experience, using the Kahoot! tool, in eight undergraduate subjects related to business management, during the 2018-2019 academic year. We carried out a statistical analysis to identify the average, maximum and minimum scores of the students in the different tests and to relate them to their academic results in the first official examination call. The results show that the subject's final mark is determined by the average mark obtained on the Kahoot! test, the percentage of tests taken and the subject in question.
\end{abstract}

Keywords: gamification; Kahoot!; academic performance.

\section{Resumen}

La utilización en el aula de herramientas metodológicas más activas y participativas es una tendencia al alza en cualquier universidad. Además, observamos como es cada vez más habitual el uso de los smartphones o tablets que permiten aplicar la gamificación con los estudiantes mediante aplicaciones como Kahoot!. En este sentido, varios trabajos han mostrado algunos efectos positivos de esta herramienta, tales como la mejora del interés, la atención y la motivación del alumnado. Sin embargo, son pocos los estudios que han analizado si el uso de esta herramienta Kahoot! influye positivamente en el rendimiento académico de los estudiantes.

En este trabajo presentamos los resultados de una experiencia de gamificación, utilizando la herramienta Kahoot!, en ocho asignaturas de Grado relacionadas con la gestión empresarial, durante el curso académico 2018-2019. Realizamos un análisis estadístico para identificar las calificaciones medias, máximas y mínimas de los estudiantes en los diferentes tests y relacionarlas con sus resultados académicos en la primera convocatoria oficial de examen. Los resultados muestran que la calificación final de la asignatura está influenciada por la calificación media obtenida en el Kahoot!, el porcentaje de tests realizados y la asignatura de que se trate.

Palabras clave: gamificación; Kahoot!;rendimiento académico. 


\section{Introducción}

El nuevo marco para el aprendizaje en los diferentes niveles académicos y, especialmente, en la educación superior, está orientado hacia un aprendizaje más activo, centrado en el alumno, que incluye varias formas de aprendizaje y que es capaz de recompensar el logro de las competencias por parte de los estudiantes, tanto dentro como fuera de los programas de estudios. En este contexto, el "Informe Horizon 2017" (Adams et al., 2017) incluye las tendencias y recomendaciones en un corto período de tiempo, así como el diseño de un aprendizaje mixto y colaborativo o el proceso de aprendizaje basado en el uso de smartphones (m-learning) utilizando las nuevas tecnologías.

La expansión de las nuevas tecnologías requiere cambios significativos en el sistema educativo (Glowacki et al., 2018). El avance tecnológico y su progreso continuo han transformado la forma en la que las actividades se realizan a diario. La evolución de las tecnologías de la información junto con la creciente velocidad de las comunicaciones basadas en Internet ha promovido el uso de software de simulación y juegos para enriquecer la experiencia de aprendizaje en varias áreas (Juan et al., 2017).

En el contexto de la educación universitaria, los educadores ahora tienen la oportunidad de introducir e integrar en su formación, a través de la tecnología, actividades de aprendizaje basadas en juegos (Tan et al., 2018). El uso de la tecnología (ordenadores, teléfonos inteligentes y tabletas) se ha demostrado que fomenta y refuerza el aprendizaje, mejorando el compromiso de los estudiantes y la participación activa en las aulas. Este uso también es, sin duda, de un gran interés para el profesorado en términos de ayuda para lograr el aumento de la motivación, así como del nivel de participación de los estudiantes en la clase. Además, es útil para evaluar la comprensión y el desarrollo general de los estudiantes. Finalmente, otra ventaja es que los estudiantes también pueden disfrutar de la oportunidad de participar en su aprendizaje y controlar su propio progreso y comprensión (Koile y Singer, 2008).

En los últimos años, una de las áreas de desarrollo, en este sentido, es el uso de la gamificación y su implementación en la educación superior a través de la mejora de las plataformas educativas existentes y de la creación de nuevos programas de aprendizaje basados en juegos para su utilización en entornos de información y educación abiertos (Glowacki et al., 2018). La gamificación en educación se refiere a la introducción de elementos de juego y experiencias lúdicas en el diseño de procesos de aprendizaje. Se ha adoptado para apoyar el aprendizaje en una variedad de contextos y áreas temáticas y para abordar actitudes, actividades y comportamientos relacionados, como enfoques participativos, colaboración, estudio autoguiado, realización de tareas, hacer que las evaluaciones sean más fáciles y efectivas, integración de enfoques exploratorios para aprender y fortalecer la creatividad y retención de los estudiantes (Caponetto et al., 2014).

Así, la combinación de la gamificación y las nuevas tecnologías parece ofrecer a los docentes nuevas posibilidades para el proceso de enseñanza-aprendizaje que, en muchos casos, ya se están asumiendo como una fuente de innovación.

En este contexto, aunque hay varias aplicaciones (Socrative, Quizizz, Googleforms, Brainscape, Cerebriti, etc.) que favorecen la transición hacia metodologías de aprendizaje más activas e innovadoras, Kahoot! es una aplicación gratuita que ha aumentado su popularidad entre los docentes gracias a su facilidad de uso y a su capacidad para crear una dinámica activa en el aula (Rodríguez, 2017). Además, esta herramienta tiene varias características que la hacen adecuada como herramienta de aprendizaje y evaluación (Wang y Lieberoth, 2016).

En este trabajo nos centramos en la introducción de nuevas tecnologías y de la gamificación mediante el uso de la aplicación Kahoot!, siendo el objetivo principal analizar la relación entre el uso de esta 
herramienta de gamificación y los resultados de aprendizaje de los estudiantes en el ámbito de la educación superior, medidos a través del rendimiento académico.

El trabajo está estructurado de la siguiente manera: a continuación presentamos los principales fundamentos teóricos y prácticos de la gamificación en la educación superior y la introducción de la aplicación Kahoot! en ese ámbito. En esta parte, además, se presentan las principales hipótesis para este trabajo. En el siguiente apartado, explicamos la metodología que se ha seguido en el trabajo. Y, por último, se incluyen las conclusiones principales y las referencias que se han utilizado.

\section{Objetivos e hipótesis de investigación}

La gamificación se define como "el uso de elementos de diseño de un juego y el pensamiento del juego en contextos ajenos al juego" (Deterding et al., 2011). De esta forma, los diseñadores de juegos se sirven de las propiedades motivadoras de estos y las aplican en otras actividades de aprendizaje, captando la atención de los estudiantes. La gamificación implica compromiso y esta característica es importante en el ámbito de la educación superior para los estudiantes. Además, cada vez que el sistema de gamificación coloca a los usuarios como elemento clave en su diseño, puede facilitar el desarrollo de un entorno de aprendizaje centrado en el alumno, lo que conduce a resultados deseables (Tsay et al., 2018). Esto se debe a que el sistema de educación superior, como sistema social, requiere hacer frente al entorno en el que opera. Es necesario tener en cuenta todo lo que ha cambiado en el entorno en el que actúan las universidades, especialmente en el contexto de la cuarta revolución industrial (Simionescu, 2017) y con la nueva generación de estudiantes (los millennials).

En la última década, esta tecnología innovadora se ha implementado en la educación tratando de aumentar la participación de los estudiantes en las actividades del aula y hacer que el proceso de aprendizaje sea más atractivo para ellos. La gamificación en la educación superior influye en el comportamiento de los estudiantes, aumentando su compromiso y motivación $\mathrm{y}$, por lo tanto, ayudándolos a mejorar su conocimiento y sus habilidades. Para ello, hace uso de características tradicionales de juegos como premios o programas de fidelización (Glowacki et al., 2018). En este sentido, la gamificación en la educación superior promete una mayor motivación y autonomía, pero también puede proporcionar una sorpresa para el estudiante debido al cambio de paradigma pedagógico, ya que no a todos los estudiantes les gusta jugar. De todos modos, la gamificación es una oportunidad para proporcionar experiencias ricas y motivadoras que pueden aumentar la participación de los estudiantes y, por lo tanto, su profundidad de aprendizaje (Lopes \& Mesquita, 2015).

Actualmente, involucrar a los estudiantes en el aprendizaje se está convirtiendo en una prioridad para muchos educadores y desde muchos enfoques. La gamificación educativa puede ser un medio para ofrecer un entorno de aprendizaje centrado en el usuario, autónomo y flexible, alentando a sus usuarios a perseguir sus propios objetivos y participando en actividades de nivel más profundo de manera más persistente. Implica la adopción de propiedades motivadoras de los juegos en la enseñanza y el aprendizaje, considerando el deseo humano de comunicarse y compartir los logros como un medio para motivar a los estudiantes a aprender. Incluso si esto tiene éxito, puede resultar un compromiso a largo plazo y más profundo entre los alumnos (Tsay et al., 2018).

Por lo tanto, la presencia de la tecnología en las aulas ha inspirado un cambio de las clases tradicionales a los entornos de aprendizaje digital integrado. Estos entornos de aprendizaje interactivos presentan la oportunidad de evolucionar en el proceso de enseñanza mediante la incorporación de elementos del juego que han demostrado captar la atención del usuario, motivar hacia los objetivos y promover la competencia, el trabajo en equipo efectivo y la comunicación (Subhash y Cudney, 2018). En este sentido, 
herramientas de aprendizaje basadas en juegos como Kahoot! complementan las prácticas pedagógicas con nuevas soluciones tecnológicas.

Juegos como Kahoot! son canales para evaluar si se han logrado los objetivos de aprendizaje. Kahoot! (https://getkahoot.com) es una plataforma en línea que fue desarrollada en 2013 por el profesor Alf Inge Wang (Universidad Noruega de Ciencia y Tecnología). Es una plataforma gratuita que brinda a los profesores la oportunidad de crear sus propios cuestionarios y encuestas o usar algunos de los existentes en un banco de tests que se ponen a disposición de todos. Los resultados se muestran al final de cada juego y el profesor puede guardar la información en un documento digital. En cuanto a los estudiantes (jugadores), no están obligados a registrarse para realizar un Kahoot!, para ello se les proporciona un código del juego para unirse a un grupo que ha generado el profesor (anfitrión del juego) en https://kahoot.it/\#/ (Tan et al., 2018).

En Kahoot!, como un juego digital de respuesta estudiantil, el profesor y los estudiantes en el aula pueden interactuar a través de juegos de conocimiento competitivos utilizando la infraestructura del mismo (Tan et al., 2018). Autores como Wang et al. (2016) señalaron que Kahoot! representa una nueva generación de sistemas de respuesta estudiantil que se enfoca en la motivación y el compromiso del estudiante a través de la gamificación. Esta plataforma es apta para incrementar la motivación y el compromiso (que promueve el aprendizaje), y para evaluar la comprensión de diferentes contenidos académicos por parte de los estudiantes.

\subsection{Gamificación en educación superior y resultados}

La revisión sistemática del aprendizaje gamificado en la educación superior revela una serie de hallazgos clave que muestran que la influencia y aceptación de la gamificación y el aprendizaje basado en juegos en la educación está creciendo. La investigación en este campo ha aumentado en los últimos años con beneficios en entornos de educación superior cada vez más establecidos y reconocidos. El aprendizaje gamificado en la educación superior no recibió atención hasta 2013, pero desde entonces ha crecido rápidamente. La implementación exitosa de la gamificación y el aprendizaje basado en juegos dan razones para interesarse por su aplicación en la educación superior en diferentes culturas, materias y formatos de países / estudiantes. La revisión sistemática de la literatura identificó varios beneficios del uso del aprendizaje gamificado, como una mejor participación del estudiante, una mayor motivación, mayor confianza, mejor actitud, un incremento en el aprendizaje percibido y un mayor rendimiento. La mejora de las actitudes, el compromiso y el rendimiento de los estudiantes fueron los beneficios más significativos del uso de aplicaciones de aprendizaje basado en juegos y gamificación (Subhash \& Cudney, 2018).

En este sentido, varios estudios empíricos consideran que el uso de la gamificación en la educación superior ha crecido con resultados positivos (Dicheva et al., 2015; Wiggins, 2016) y con mejoras en asistencia, participación y motivación de los estudiantes (Barata et al., 2013; Mitchell et al., 2013; O'Donovan et al., 2013). Además, el uso de la gamificación se ha extendido en algunos campos de aprendizaje, como la gestión empresarial y el marketing. A pesar de esto, otros estudios empíricos concluyen con la preocupación de que la gamificación conduzca a una complejidad excesiva o una competencia excesiva (Berkling y Thomas, 2013; De-Marcos et al., 2014; Haaranen et al., 2014), creando diferentes 'estatus' de estudiantes: aquellos proactivos y comprometidos que siempre ganan (triunfadores), aquellos que comienzan con fuerza y pierden interés en el camino (desanimados) y aquellos con bajos niveles de participación, menos compromiso y peor desempeño (bajo rendimiento) (Hung, 2017).

Las diferentes experiencias innovadoras implementadas en la educación superior coinciden en incorporar los ingredientes de los escenarios recreativos, proponiendo a los estudiantes la resolución de problemas, 
la elaboración de proyectos y la realización de diversas misiones o actividades siguiendo ciertos hitos (Fitz-Walter et al., 2011). En otras palabras, los estudiantes deben adoptar los mecanismos de los juegos convencionales y establecer niveles que deben lograrse junto con la asignación de puntos a cada desafío que se complete con éxito (O'Donovan et al., 2013). Estas experiencias garantizan un aumento de la motivación extrínseca e intrínseca de los estudiantes y fomentan la inmersión en las tareas propuestas (Hamari et al., 2014; Papasterigou, 2009).

En última instancia, se espera que estas experiencias ayuden a los alumnos a desarrollar habilidades laborales y a abordar las necesidades psicológicas de habilidades, independencia y relaciones y, por lo tanto, a aumentar la motivación de los estudiantes en contextos de trabajo o aprendizaje (Sailer et al., 2017; Tsihouridis et al., 2018).

Los estudios empíricos concluyen que Kahoot! es una buena herramienta para implementarse en el aula, que contribuye a aumentar la participación de los estudiantes, quienes la perciben como un juego. Además, conduce a mejorar la relación social entre los estudiantes, aumentando también la asistencia a las clases.

La mayoría de los estudios mencionados anteriormente relacionan la gamificación en la educación superior con la valoración que realizan los estudiantes sobre la motivación, la cooperación, el compromiso, la competencia, la asistencia, la participación, etc. Sin embargo, hasta este momento no existe investigación en el campo de la educación superior que relacione la gamificación y el uso de Kahoot! con los resultados académicos de los estudiantes en el área de Administración de Empresas. Monedero y Castro (2018) indican que cuando los estudiantes aprenden jugando están más satisfechos, retienen mejor lo que han aprendido y también se sienten atraídos por continuar aprendiendo. En este contexto, nuestro objetivo se centra en la evaluación de si el uso de esta herramienta, además de generar una dinámica más participativa en las clases, permite a los estudiantes retener mejor lo que han aprendido, reflejándose así en mejores resultados académicos. Proponemos, por tanto, la siguiente hipótesis de investigación:

H1: Existe una relación positiva entre los resultados que se obtienen en las pruebas realizadas en Kahoot! y la nota final que se obtiene en la asignatura.

\section{Desarrollo de la innovación}

Esta experiencia de utilización de Kahoot! como herramienta de evaluación de contenidos ha sido utilizada en ocho asignaturas diferentes relacionadas con la gestión de empresas y que se han impartido en diferentes Grados a lo largo de los dos semestres del curso 2018-2019.

En todas las asignaturas se ha realizado un Kahoot! por tema o unidad, al final del mismo, que sirviera para la comprobación de los conceptos asimilados por los estudiantes. Todas las preguntas han sido elaboradas por las profesoras responsables de las asignaturas y estaban relacionadas con los contenidos abordados en cada unidad. En la gran mayoría de los casos (excepto cuando había algunas preguntas repetidas sobre el mismo aspecto), al finalizar el tiempo de respuesta y, tras comprobar los fallos que, en su caso hubiera, la profesora se paraba a explicar cuál era la respuesta correcta o pedía a alguno de los compañeros que lo explicara.

Se ha trabajado con grupos relativamente reducidos y esto ha facilitado mucho la puesta en práctica de esta herramienta. Concretamente, los estudiantes que han trabajado en el total de estas asignaturas han sido 286. El reparto de la población entre asignaturas y titulaciones aparece reflejado en la tabla 1 y en los Gráficos 1 y 2 . 
Tabla 1. Distribución de estudiantes por titulación y asignatura

\begin{tabular}{|c|c|c|c|}
\hline Titulación & Asignatura & Curso & $\mathrm{N}^{\mathrm{o}}$ de estudiantes \\
\hline \multirow{3}{*}{$\begin{array}{l}\text { Grado en Administración y } \\
\text { Dirección de Empresas (ADE) }\end{array}$} & Dirección Estratégica II & $4^{\circ}$ & 43 \\
\hline & Dirección Financiera II & $3^{\circ}$ & 63 \\
\hline & $\begin{array}{l}\text { Dirección y administración de la } \\
\text { producción }\end{array}$ & $3^{\circ}$ & 53 \\
\hline $\begin{array}{l}\text { Doble Grado en Derecho y } \\
\text { Administración y Dirección de } \\
\text { Empresas (DADE) }\end{array}$ & Dirección Estratégica II & $5^{\circ}$ & 26 \\
\hline $\begin{array}{l}\text { Grado en Estadística } \quad \text { y } \\
\text { Empresa (EyE) }\end{array}$ & $\begin{array}{l}\text { Gestión estratégica de } \\
\text { innovación, la la calidad y } \\
\text { tecnología }\end{array}$ & $4^{\circ}$ & 15 \\
\hline \multirow{2}{*}{$\begin{array}{l}\text { Grado en Finanzas } \quad \text { y } \\
\text { Contabilidad (FICO) }\end{array}$} & Contabilidad de Gestión I & $2^{\circ}$ & 23 \\
\hline & Gestión de Recursos Humanos & $2^{\circ}$ & 28 \\
\hline $\begin{array}{l}\text { Grado en Relaciones Laborales } \\
\text { y Recursos Humanos (RRLL) }\end{array}$ & $\begin{array}{l}\text { Organización y Administración de } \\
\text { empresas }\end{array}$ & $1^{\mathrm{o}}$ & 35 \\
\hline
\end{tabular}

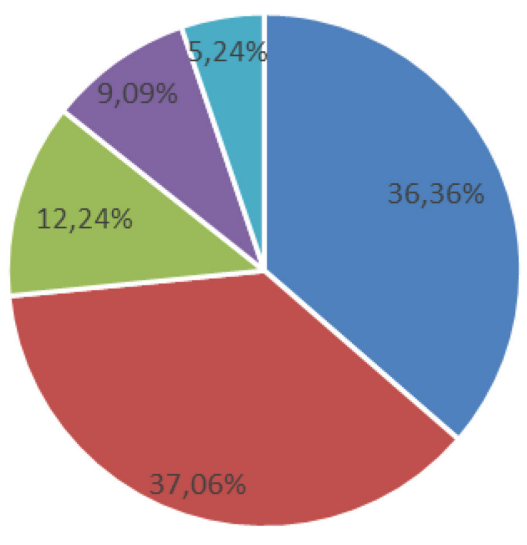

- Grado en Finanzas y Contabilidad

- Grado en Administración y Dirección de Empresas

- Grado en Relaciones Laborales y Recursos Humanos

- Doble Grado Derecho y ADE

- Grado en Estadística y Empresa

Gráfico 1: Distribución de estudiantes por titulación 


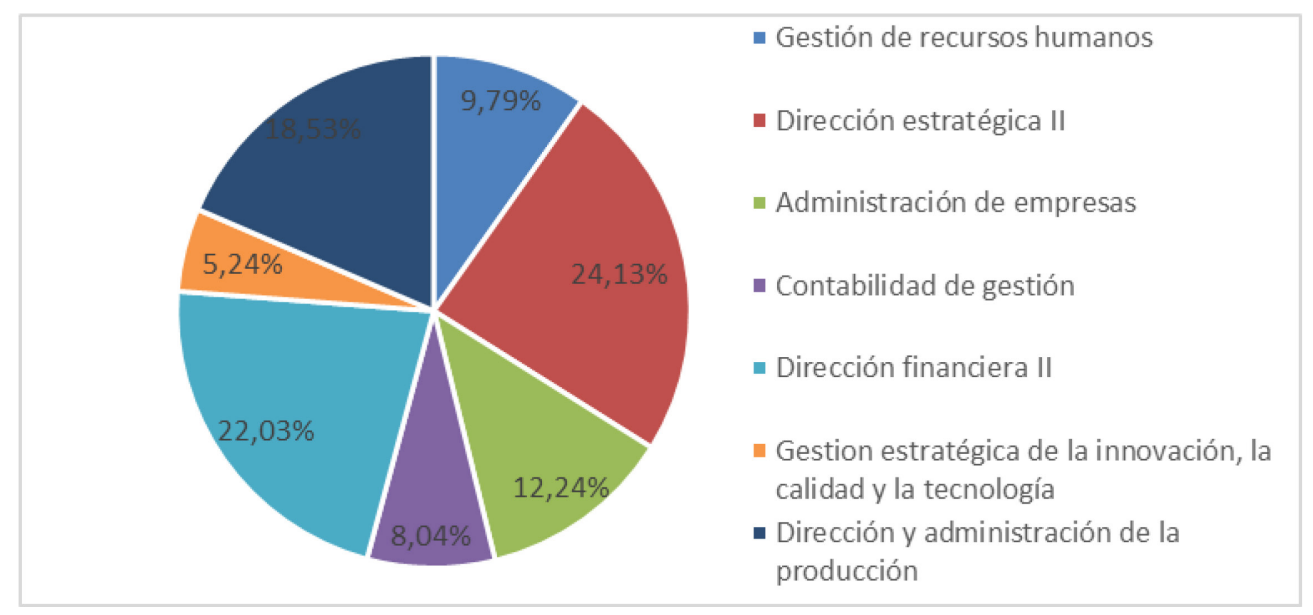

Gráfico 2: Distribución de estudiantes por asignatura

No en todas las asignaturas se ha trabajado con el mismo número de pruebas Kahoots puesto que, como decíamos, dependía del número de unidades o temas a evaluar, pero sí se han realizado siempre tests que incluían 10 preguntas, para así facilitar la valoración de los resultados obtenidos en los mismos.

Hay que tener en cuenta también que en cinco asignaturas (Gestión de recursos humanos de FICO; Organización y administración de empresas de RRLL; Contabilidad de gestión I; Dirección financiera II y Gestión estratégica de la innovación, la calidad y la tecnología de ADE) los resultados obtenidos en los test de Kahoot! se tenían en cuenta en la calificación final de la asignatura como parte de una evaluación sumativa-formativa, incluyéndolos en el apartado de asistencia y participación. Sin embargo, en las otras tres asignaturas no se han tenido en cuenta las calificaciones obtenidas en los Kahoots para la calificación final, sirviendo únicamente como elemento de análisis individualizado de cada estudiante por parte de la profesora.

El análisis de los datos se realizó con el programa estadístico SPSS Statistics versión 19 y se corresponde con un análisis estadístico descriptivo (frecuencias, medias, desviaciones típicas, ec.) de las calificaciones obtenidas en los Kahoot! realizados. Además, hemos realizado tablas de contingencia con pruebas de Chicuadrado, así como un análisis de regresión para determinar si existe relación o no entre las calificaciones obtenidas en los Kahoot! y las calificaciones obtenidas en la asignatura.

\section{Resultados}

En primer lugar, vamos a hacer referencia al número de pruebas Kahoots realizados en cada asignatura y el número de estudiantes que participaron en los distintos Kahoots. Así, en las asignaturas de Dirección Estratégica II (tanto en ADE como en DADE) fue donde más tests se realizaron; concretamente, se hicieron 7 Kahoots. Le sigue Gestión de Recursos Humanos de FICO con 6 tests. En Organización y Administración de Empresas de RRLL se realizaron 5 Kahoots a lo largo del semestre. Tres asignaturas (Contabilidad de Gestión I de FICO, Gestión estratégica de la innovación, la calidad y la tecnología de EyE y Dirección y administración de la producción de ADE) completaron 4 Kahoots cada una de ellas. Finalmente, en Direccion Financiera II de ADE solamente se desarrollaron dos tests, puesto que el programa de esta asignatura contempla dos grandes bloques. 
Por lo que respecta al grado de participación de los estudiantes en estos tests, tal y como se puede observar en el gráfico 3 y en la tabla 2, donde reflejamos el porcentaje de estudiantes en función del porcentaje de Kahoots completados, la asignatura que ha tenido un mayor porcentaje de estudiantes que han asistido con más regularidad a clase y que se han implicado más en la realización de los distintos Kahoots han sido Gestión estratégica de la innovación, la calidad y la tecnología. Esta asignatura es una optativa del grado de Estadística y Empresa, lo que significa que los estudiantes eligen voluntariamente cursarla y eso puede explicar la gran participación y asistencia a clase. A continuación, nos encontraríamos la asignatura Dirección Financiera II de ADE, aunque en este caso habría que ser un poco cautelosos con el análisis puesto que sólo se han realizado dos Kahoots en esa asignatura. Finalmente, como más representativo, podemos destacar el grupo de $2^{\circ}$ del Grado en FICO que, tanto en la asignatura de Gestión de Recursos Humanos (primer semestre) como en la Contabilidad de Gestión I (segundo semestre) han mostrado un alto índice de asistencia a clase y de participación en los Kahoots realizados.

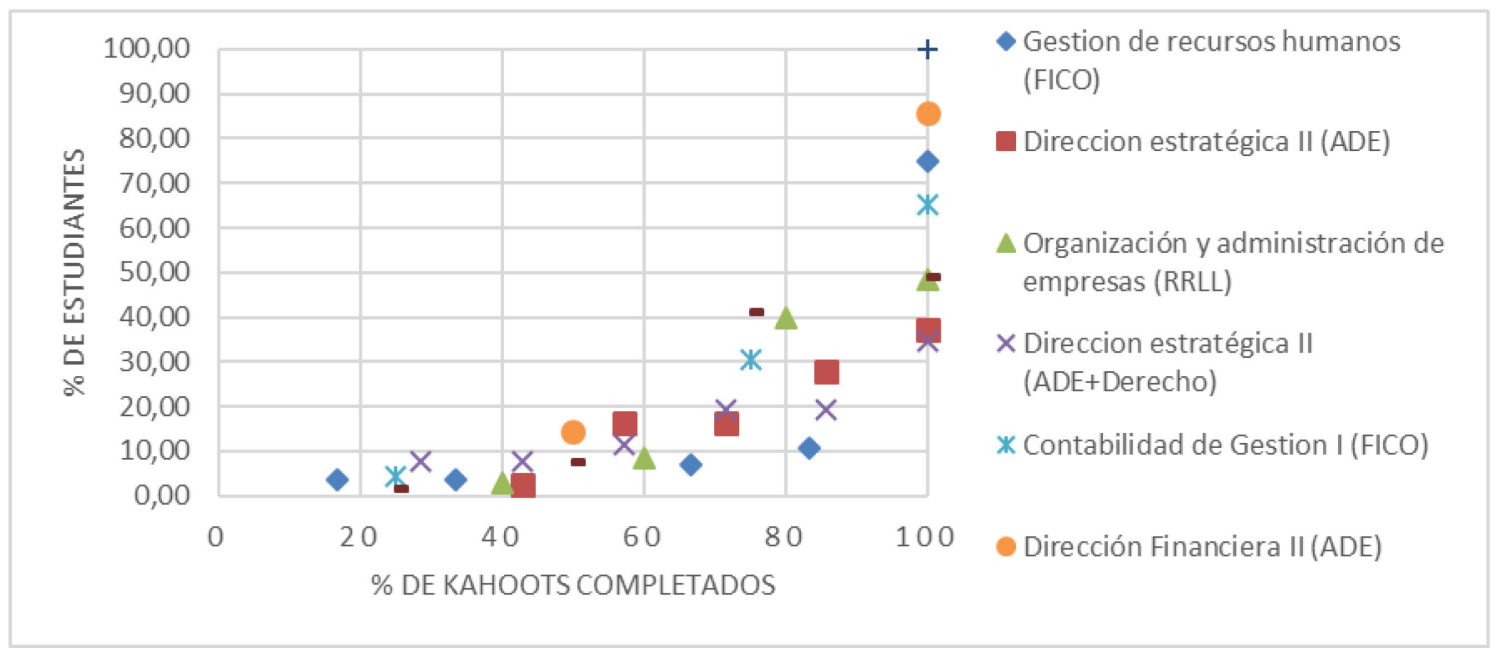

Gráfico 3. \% de estudiantes $y \%$ de Kahoots realizados

Tabla 2. Porcentaje de estudiantes en función del grado de participación en los Kahoots

\begin{tabular}{|l|c|c|c|c|c|c|c|}
\hline \multicolumn{1}{|c|}{ Asignatura } & 7 & 6 & 5 & 4 & 3 & 2 & 1 \\
\hline $\begin{array}{l}\text { Dirección estratégica II de ADE } \\
\text { (7 Kahoots) }\end{array}$ & 37,21 & 27.90 & 16,28 & 16,28 & 2,32 & 0 & 0 \\
\hline $\begin{array}{l}\text { Dirección estratégica II de DADE } \\
\text { (7 Kahoots) }\end{array}$ & 34,61 & 19,23 & 19,23 & 11,54 & 7,69 & 7,69 & 0 \\
\hline $\begin{array}{l}\text { Gestión de Recursos Humanos de } \\
\text { FICO (6 Kahoots) }\end{array}$ & - & 75.00 & 10,71 & 7,15 & 0 & 3.57 & 3,57 \\
\hline $\begin{array}{l}\text { Organización y administración de } \\
\text { empresas de RRLL (5 Kahoots) }\end{array}$ & - & - & 48,57 & 40,00 & 8,57 & 2,86 & 0 \\
\hline $\begin{array}{l}\text { Contabilidad de Gestión I de FICO } \\
\text { (4 Kahoots) }\end{array}$ & - & - & - & 65.22 & 30.43 & 0 & 4.35 \\
\hline $\begin{array}{l}\text { Gestión estratégica de la innovación, } \\
\text { la calidad y la tecnología de EyE } \\
\text { (4 Kahoots) }\end{array}$ & - & - & - & 100 & 0 & 0 & 0 \\
\hline $\begin{array}{l}\text { Dirección y administración de la } \\
\text { producción de ADE (4 Kahoots) }\end{array}$ & - & - & - & 49.06 & 41.51 & 7.55 & 1.89 \\
\hline $\begin{array}{l}\text { Dirección Financiera II de ADE } \\
\text { (2 Kahoots) }\end{array}$ & - & - & - & - & - & 85.71 & 14.29 \\
\hline
\end{tabular}


Por lo que respecta a las calificaciones obtenidas, como ya comentamos anteriormente, los Kahoots incluían 10 preguntas y eran valorados sobre 10 puntos para facilitar tanto al estudiante como a las profesoras su tratamiento y consideración para la calificación final. Esto implica que, al margen de la valoración en puntos que concede automáticamente el programa (en función de si se acierta o no la respuesta y del tiempo que se tarda en responder), las profesoras lo que tenían en consideración para la evaluación era únicamente el número de respuestas acertadas. Así, pues, en la siguiente tabla, mostramos para cada asignatura, cuál ha sido la calificación media obtenida por los estudiantes en los distintos Kahoots, así como la puntuación máxima y la mínima.

Tabla 3. Calificaciones medias, máximas y mínimas de los Kahoots realizados

\begin{tabular}{|c|c|c|c|}
\hline Calificación Kahoot! & $\begin{array}{l}\text { Calificación } \\
\text { media }\end{array}$ & $\begin{array}{l}\text { Calificación } \\
\text { mínima }\end{array}$ & $\begin{array}{l}\text { Calificación } \\
\text { máxima }\end{array}$ \\
\hline Gestión de Recursos Humanos de FICO & 8.02 & 3 & 10 \\
\hline Dirección Estratégica II de ADE & 3.78 & 1 & 10 \\
\hline Organización y Administración de Empresas de RRLL & 5.51 & 1 & 9 \\
\hline Dirección Estratégica II de DADE & 5.54 & 2 & 10 \\
\hline Contabilidad de Gestión I de FICO & 4.96 & 1 & 8 \\
\hline Dirección Financiera II de ADE & 4.83 & 1 & 8 \\
\hline $\begin{array}{l}\text { Gestión estratégica de la innovación, la calidad y la } \\
\text { tecnología de EyE }\end{array}$ & 8.13 & 4 & 10 \\
\hline Dirección y administración de la producción de ADE & 6.77 & 1 & 10 \\
\hline
\end{tabular}

Como se puede comprobar en esta tabla, los estudiantes de Dirección estratégica II de ADE son los que han obtenido la media de calificaciones más bajas. Creemos que en ello puede influir el hecho de que esta era una de las asignaturas en las que no se tenían en cuenta los resultados de los Kahoots para la calificación final de la asignatura, por lo que podríamos decir que los estudiantes simplemente participaban sin preocuparse demasiado por si acertaban o no. No obstante, puesto que esto no ocurre en todas las asignaturas en las que no se tenía en cuenta la calificación, creemos que sería recomendable analizar esta información considerando la dificultad o facilidad tanto de la propia asignatura como de las preguntas realizadas por las profesoras o el grado de conocimiento o preparación previa de los estudiantes, cuestiones éstas mucho más subjetivas y difíciles de abordar.

Otra forma de presentar y analizar las calificaciones medias obtenidas por los estudiantes en los diferentes Kahoots es agrupándolas en las categorías de Suspenso (0-4.9); Aprobado (5-6.9); Notable (7-8.9) y Sobresaliente (9-10). Así, en la tabla 4 presentamos el número total de estudiantes cuya calificación media de los diferentes Kahoots se sitúa en cada una de las categorías. 
Tabla 4. Número de estudiantes según su calificación media en los Kahoots

\begin{tabular}{|l|l|l|l|l|l|}
\hline Calificación Kahoot! & Suspenso & Aprobado & Notable & Sobresaliente & TOTAL \\
\hline Gestión de Recursos Humanos de FICO & 0 & 1 & 22 & 5 & 28 \\
\hline Dirección Estratégica II de ADE & 38 & 5 & 0 & 0 & 43 \\
\hline $\begin{array}{l}\text { Organización y Administración de } \\
\text { Empresas de RRLL }\end{array}$ & 10 & 23 & 2 & 0 & 35 \\
\hline Dirección Estratégica II de DADE & 7 & 17 & 2 & 0 & 26 \\
\hline Contabilidad de Gestión I de FICO & 10 & 13 & 0 & 0 & 23 \\
\hline Dirección Financiera II de ADE & 30 & 30 & 3 & 0 & 63 \\
\hline $\begin{array}{l}\text { Gestión estratégica de la innovación, la } \\
\text { calidad y la tecnología de EyE }\end{array}$ & 0 & 0 & 13 & 2 & 15 \\
\hline $\begin{array}{l}\text { Dirección y administración de la } \\
\text { producción de ADE }\end{array}$ & 11 & 19 & 14 & 9 & 53 \\
\hline
\end{tabular}

En este caso se observa que los estudiantes de $3^{\circ}$ y $4^{\circ}$ curso del Grado en ADE, tanto en la asignatura Dirección Financiera II como en Dirección Estratégica II, son los que más suspenden los Kahoots, probablemente debido a que la dificultad de las asignaturas y/o de las preguntas es mayor. Por el contrario, las asignaturas donde los estudiantes han obtenido las mejores calificaciones han sido Gestión estratégica de la innovación, la calidad y la tecnología de EyE y Gestión de Recursos Humanos de FICO.

Teniendo en cuenta estas calificaciones, tal y como comentamos en el apartado teórico, quisimos analizar si existe relación entre las calificaciones obtenidas por los estudiantes en los Kahoots y la calificación obtenida en la asignatura en la convocatoria ordinaria I. Si esta relación fuera positiva y estadísticamente significativamente, estaríamos asumiendo que aquellos estudiantes que mejor calificación obtienen en los Kahoots durante el semestre, serían también aquellos que mejores calificaciones obtienen al final en la asignatura.

Para contrastar esta hipótesis, hemos realizado una tabla de contingencia con el análisis estadístico de la Chi-cuadrado. Los resultados aparecen reflejados en las tablas 5 y 6 . 
Tabla 5. Tabla de contingencia Calificación media de los Kahoots * Calificación ordinaria

\begin{tabular}{|l|l|c|c|c|c|c|}
\hline \multicolumn{2}{|c|}{} & \multicolumn{3}{|c|}{ Calificación asignatura Convocatoria Ordinaria } & \\
\cline { 3 - 7 } \multicolumn{2}{|c|}{} & Suspenso & Aprobado & Notable & Sobresaliente & TOTAL \\
\hline \multirow{2}{*}{$\begin{array}{l}\text { Calificación } \\
\text { media }\end{array}$} & Suspenso & 34 & 41 & 20 & 11 & 106 \\
\cline { 2 - 7 } Kahoots & Aprobado & 16 & 33 & 35 & 24 & 108 \\
\cline { 2 - 7 } & Notable & 2 & 11 & 34 & 9 & 56 \\
\cline { 2 - 7 } & Sobresaliente & 0 & 1 & 3 & 12 & 16 \\
\hline
\end{tabular}

Tabla 6. Prueba de chi-cuadrado

\begin{tabular}{|l|l|l|l|}
\hline & Valor & Gl & Sig. Asintótica (bilateral) \\
\hline Chi-cuadrado de Pearson & 80,408 & 9 & 0,000 \\
\hline Razón de verosimilitudes & 74,650 & 9 & 0,000 \\
\hline Asociación lineal por líneas & 49,177 & 1 & 0,000 \\
\hline N de casos válidos & 286 & & \\
\hline
\end{tabular}

Como podemos comprobar, la relación entre ambas variables es completamente significativa desde el punto de vista estadístico al 99.9\%, observándose, como era de esperar, que la mayoría de los estudiantes que suspenden los Kahoots obtienen la calificación de suspenso o aprobado y que aquellos estudiantes que han sacado un sobresaliente en los Kahoots han obtenido, en una gran proporción, un sobresaliente en la asignatura. También se observan algunos casos un poco más chocantes como, por ejemplo, estudiantes que habiendo obtenido un notable en los Kahoots han suspendido la asignatura o estudiantes que habiendo obtenido un aprobado en los Kahoots han obtenido sobresaliente en la asignatura. Aun así, podemos afirmar que se cumple nuestra hipótesis 1.

Pero, además de la calificación media obtenida en los Kahoots, nos preguntamos si podían influir también otras variables como, por ejemplo, el porcentaje de Kahoots resueltos y la asignatura de que se tratara. En este caso, realizamos un análisis de regresión por pasos donde la variable dependiente es la calificación obtenida en la convocatoria ordinaria 1. Como presentamos en la tabla 7, el modelo final presenta una $\mathrm{R}$ cuadrado corregida de 0.321 lo que significa que el modelo es relativamente bueno y en todos los casos se obtiene una significación de 0.000 .

Tabla 7. Resultados del análisis de regresión para las variables predictoras del rendimiento académico durante el curso 2018-2019 $(n=286)$

\begin{tabular}{|l|l|l|l|}
\hline Variable & Paso 1 & Paso 2 & Paso 3 \\
\hline Media Kahoot! & $.627^{* * * *}$ & $.565^{* * *}$ & $.536^{* * *}$ \\
\hline \% de Kahoots & & $.030^{* * *}$ & $.029^{* * *}$ \\
\hline Asignatura & & & $.199^{* * *}$ \\
\hline $\mathrm{F}$ & $86.347^{* * *}$ & $57.027^{* * *}$ & $45.902^{* * *}$ \\
\hline $\mathrm{R}^{2}$ ajustado & .230 & .282 & .321 \\
\hline Cambios en $\mathrm{R}^{2}(\%)$ & & 22.61 & 13.83 \\
\hline
\end{tabular}

Nota: ${ }^{* * *} \mathrm{p}<0.001$ 
Por tanto, tal y como demuestra el modelo, podemos decir que la calificación que los estudiantes obtienen en la convocatoria ordinaria viene explicada por el \% de Kahoots que realizan, por la calificación media que obtienen en estos test y por la asignatura de que se trate, siendo todas las relaciones positivas y estadísticamente significativas.

\section{Conclusiones}

La gamificación con Kahoot! ha sido una herramienta de enseñanza innovadora en nuestras materias, tanto para los profesores como para los estudiantes. Esta experiencia ha permitido aprovechar al máximo las nuevas tecnologías para la enseñanza con resultados más que aceptables en términos de implicación, participación, interés y motivación, de acuerdo con estudios previos en otros campos de investigación (Signori et al., 2018). No hay duda sobre los efectos emocionales positivos de la gamificación (Caponetto et al., 2014). Sin embargo, nuestro objetivo ha ido más allá, tratando de comprobar si existe una relación significativa entre el uso de la gamificación con Kahoot! como herramienta de evaluación y los resultados académicos de los estudiantes de los diferentes grados relacionados con la Administración de Empresas.

En la hipótesis, en la que indicamos la posibilidad de que los resultados académicos mejoren en aquellos alumnos que han tenido mejor calificación en los kahoots realizados, verificamos que, aunque no todas las asignaturas tienen el mismo comportamiento, en la mayoría de ellas se puede observar una mejor calificación final de los estudiantes y un menor número de calificaciones suspensas.

Además de estos resultados del estudio, nos gustaría destacar algunas consideraciones que, aunque podrían ser algo subjetivas, creemos que son interesantes. En primer lugar, Kahoot! se ha demostrado que es una herramienta útil que ha mejorado la asistencia y participación de nuestros estudiantes. Esta herramienta de aprendizaje ha favorecido tanto el proceso de aprendizaje continuo como el compromiso de los estudiantes. Los estudiantes no solo asisten a más clases, sino que también estudian el tema de manera más regular para obtener mejores resultados en cada test. Aún más, Kahoot! nos permite reforzar los conceptos principales de la asignatura dando una retroalimentación inmediata a los estudiantes. Uno de los elementos más didácticos que hemos percibido ha sido la pausa después de cada pregunta, explicando la respuesta correcta. De esta manera, los estudiantes pueden hacer una autoevaluación, entendiendo los conceptos estudiados más fácilmente.

Con la utilización de la gamificación con Kahoot! hemos incorporado el m-learning, adaptando nuestros temas a las crecientes demandas tecnológicas de la generación millenials. Hemos corroborado que si los estudiantes aprenden jugando, tienden a recordar mejor los conceptos, lo que facilita el proceso de aprendizaje. Además, la dinámica del juego muchas veces conduce a un aumento en la motivación y la autoestima del estudiante, especialmente cuando ven sus nombres en el podio clasificatorio final que ofrece la aplicación.

Es importante tener en cuenta que, como hemos indicado en la metodología, en cinco asignaturas se ha seguido una evaluación sumativa-formativa, de manera que los resultados de las pruebas de Kahoot! fueron parte de la calificación final. De esta manera, los estudiantes tienen una mejor valoración sobre la utilidad de esta herramienta, especialmente si algunas de las preguntas de las pruebas también se repitieron en el examen final.

Del mismo modo, tenemos que ser conscientes de varias preocupaciones que surgen de nuestra experiencia. Hemos trabajado con un tamaño de grupos relativamente bueno. Para los grupos con un mayor número de estudiantes, los resultados no serían tan efectivos. Un mayor número de estudiantes implica mayores dificultades tanto físicas (aulas con mayor capacidad) como técnicas (acceso a la 
aplicación y fallos relacionados con el acceso). También debemos considerar la utilización responsable que requiere el uso de herramientas de gamificación durante el proceso de aprendizaje. En particular, el uso de Kahoot! se basa en la idea de que la educación basada en juegos es adecuada (Dellos, 2015) y que las apps pueden mejorar el proceso de enseñanza y aprendizaje, en función de la importancia dada a estas herramientas por los docentes (Area, 2008). Además, es necesario que la organización de las clases se base en un ámbito pedagógico bien definido, adoptando un uso responsable de estas herramientas (Marín, 2016). Bajo este uso responsable, la aplicación de herramientas de gamificación en nuestra enseñanza en educación superior ha generado un ambiente de trabajo (en términos de asistencia, compromiso y actitud del alumnado) que es favorable para la mejora del aprendizaje en el sentido de que, como hemos demostrado empíricamente, hemos conseguido una mejora de los resultados de aprendizaje de nuestros estudiantes.

\section{Referencias}

ADAMS, S., CUMMINS, M., DAVIS, A., FREEMAN, A., HALL, C., \& ANANTHANARAYANAN, V. (2017). NMC Horizon Report: 2017 Higher Education Edition. Austin, Texas.

AREA, M. (2008). Innovación pedagógica con TIC y el desarrollo de competencias informacionales y digitales. Investigación En La Escuela, 64, 5-18.

BARATA, G., GAMA, S., FONSECA, M., \& GONÇALVES, D. (2013). Improving Student Creativity with Gamificaton and Virtual Worlds. Stratford, ON, Canada: ACM.

BERKLING, K., \& THOMAS, C. (2013). Gamification of a Software Engineering course and a detailed analysis of the factors that lead to it's failure. In 2013 International Conference on Interactive Collaborative Learning (ICL) (pp. 525-530). IEEE.

CAPONETTO, I., EARP, J., \& OTT, M. (2014). Gamification and Education: A Literature Review. European Conference on Game-Based Learning, 1, 50.

DE-MARCOS, L., DOMÍNGUEZ, A., SAENZ-DE-NAVARRETE, J., \& Pagés, C. (2014). An empirical study comparing gamification and social networking on e-learning. Computers and Education, 75, 82-91. https://doi.org/10.1016/j.compedu.2014.01.012

DELLOS, R. (2015). Kahoot! A digital game resource for learning. International Journal of Institutional Technology and Distance Learning, 12(4), 49-52.

DETERDING, S., DIXON, D., KHALED, R., \& NACKE, L. (2011). From game design elements to gamefulness: defining gamification. In Proceedings of the 15th International Academic MindTreck Conference: Envisioning Future Media Environments (pp. 9-15). New York, NY.

DICHEVA, D., DICHEV, C., AGRE, G., \& ANGELOVA, G. (2015). Gamification in education: A systematic mapping study. Educational Technology \& Society, 18(3), 75-88.

FITZ-WALTER, Z., TJONDRONEGORO, D., \& WYETH, P. (2011). Orientation passport: using gamification to engage university students. In Proceedings of the 23rd Australian Computer-Human Interaction Conference (pp. 122-125).

GLOWACKI, J., KRIUKOVA, Y., \& ASHENYUK, N. (2018). Gamification in Higher Education: Experience of Poland and Ukraine. Advanced Education, 5(10), 105-110.

HAARANEN, L., HAKULINEN, L., IHANTOLA, P., \& KORHONEN, A. (2014). Software architectures for implementing achievement badges-practical experiences. In 2014 International Conference on Teaching and Learning in Computing and Engineering (pp. 41-46). IEEE.

HAMARI, J., KOIVISTO, J., \& SARSA, H. (2014). Does gamification work? A literature review of empirical studies on gamification. In 7th HI International Conference on System Sciences (HICSS) (pp. 3025-3034).

HUNG, A. (2017). A Critique and Defense of Gamification. Journal of Interactive Online Learning, 1(Summer), 5773.

JUAN, A. A., LOCH, B., DARADOUMIS, T., \& VENTURA, S. (2017). Games and simulation in higher education. Springer. 
KOILE, K., \& SINGER, D. (2008). Assessing the Impact of a Tablet-PC-based Classroom Interaction System. In R. H. Reed, D. A. Berque, \& J. C. Prey (Eds.), The Impact of Tablet PCs and Pen-based Technology on Education. Evidence and Outcomes (pp. 73-80). West Lafayette, Indiana: Purdue University Press.

LOPES, R. P., \& MESQUITA, C. (2015). Evaluation of a Gamification Methodology in Higher Education. Edulearn15: 7th International Conference on Education and New Learning Technologies, (February), 69967005.

MARÍN, D. (2016). Valoración del uso de Whatsapp en la tutorización del TFG. In I Congreso Virtual Internacional de Educación, Innovación y TIC (EDUNOVATIC) (pp. 671-673).

MITCHELL, N., DANINO, N., \& MAY, L. (2013). Motivation and manipulation: A gamification approach to influencing undergraduate attitudes in computing. In Proceedings of European Conference on Game-Based Learning (pp. 394-400).

MONEDERO, C. R., \& CASTRO, A. (2018). Un proyecto de aplicación de la clase inveertida en las Ciencias de la Comunicación. In REDINE (Ed.), Innovative Strategies for Higher Education in Spain. Eindhoven, NL: Adaya Press.

O'DONOVAN, S., GAIN, J., \& MARAIS, P. (2013). A case study in the gamificaton of a university-level games development course. In Proceedings of the South African Institute for Computer Scientists and Information Technologists Conference (pp. 242-251).

PAPASTERIGOU, M. (2009). Digital game-based learning in high school computer science education: impact on educational effectiveness and student motivation. Computers and Education, 52(1), 1-12.

RODRÍGUEZ FERNÁNDEZ, L. (2017). Smartphone y aprendizaje: el uso de Kahoot! en el aula universitaria. Revista Mediterránea de Comunicación, 8(1), 181-190.

SAILER, M., HENSE, J., MANDL, H., \& KLEVERS, M. (2017). Fostering development of work competencies and motivation via gamification. In Mudler M. (Ed.), Competence-Based Vocational and Professional Education Technical and Vocational Education and Training: Issues, Concerns and Prospects. Cham: Springer.

SIGNORI, G. G., GUIMARAES, J. C. F. DE, SEVERO, E. A., \& ROTTA, C. (2018). Gamification as an innovative method in the processes of learning in higher education institutions. International Journal of Innovation and Learning, 24(2), 115. https://doi.org/10.1504/ijil.2018.094066

SIMIONESCU, V. (2017). Using Gamification For Teaching Economics In Technical Higher Education: An Exploratory Research, (November 2018), 532-541. https://doi.org/10.15405/epsbs.2017.05.02.65

SUBHASH, S., \& CUDNEY, E. A. (2018). Gamified learning in higher education: A systematic review of the literature. Computers in Human Behavior, 87(February), 192-206. https://doi.org/10.1016/j.chb.2018.05.028

TAN, D., LIN, A., GANAPATHY, M., \& KAUR, M. (2018). Kahoot! It: Gamification in Higher Education. Pertanika J. Soc. Sci. \& Hum, 26(1), 565-582.

TSAY, C. H. H., KOFINAS, A., \& LUO, J. (2018). Enhancing student learning experience with technology-mediated gamification: An empirical study. Computers and Education, 121(April 2017), 1-17. https://doi.org/10.1016/j.compedu.2018.01.009

TSIHOURIDIS, C., VAVOUGIOS, D., \& IOANNIDIS, G. S. (2018). Assessing the learning process playing with Kahoot - A study with upper secondary school pupils learning electrical circuits. In M. Auer, D. Guralnick, \& I. Simonis (Eds.), Advances in Intelligent Systems and Computing Teaching and Learning in a Digital World (p. 175). Cham: Springer.

WANG, A. I., \& LIEBEROTH, A. (2016). The effect of points and audio concentration, engagement, enjoyment, learning, motivation, and classroom dynamics using Kahoot! Reading: Academic Conferences International LImited.

WANG, A. I., ZHU, M., \& SÆTRE, R. (2016). The effect of digitizing and gamifying quizzing in classrooms. Academic Conferences and Publishing International.

WIGGINS, B. E. (2016). An Overview and Study on the Use of Games, Simulations, and Gamification in Higher Education. International Journal of Game-Based Learning, 6(1), 18-29. https://doi.org/10.4018/ijgbl.2016010102 